\title{
Idarucizumab gets the nod as the first specific antidote for an oral anticoagulant
}

See Commentary on page 6

$\mathrm{I}$ $n$ the fall of 2015, idarucizumab became the first drugspecific antidote to an oral anticoagulant to receive regulatory approval from the US Food and Drug Administration. Anticoagulants are designed to inhibit the formation of blood clots and prevent thrombotic disorders, but they can increase bleeding risk in patients who experience trauma or illness or who undergo invasive surgical procedures. For this reason, traditional anticoagulants have antidotes that reverse their therapeutic effects, but newer oral anticoagulants lack specific reversal agents and that has proven a barrier to their use in patients with higher bleeding risk.

Idarucizumab is a monoclonal antibody fragment that binds to the direct thrombin inhibitor dabigatran and reverses its anticoagulant effect. It was approved on the basis of 3 randomized, placebo-controlled phase 1 trials in a total of 283 patients. The first was a 3-part dose-escalation study (NCT01688830) in 157 healthy male volunteers aged 18-45 years, with a body-mass index of $18.5-29.9 \mathrm{~kg} /$ $\mathrm{m}^{2}$, in which participants were treated with various doses of idarucizumab alone or in combination with dabigatran.

In part 1 of that study, involving 110 participants, each of 10 groups received a different dose of idarucizumab ranging from $20 \mathrm{mg}$ to $8 \mathrm{~g}$ - as an intravenous (IV) infusion over 1 hour, and another 3 groups received a dose of 1,2 , or $4 \mathrm{~g}$ as a 5 -minute IV infusion. ${ }^{1}$ Parts 2 and 3 of the study involved 47 participants and consisted of a risingdose assessment of idarucizumab alone $(\mathrm{n}=35)$ and a dosefinding, proof-of-concept study $(\mathrm{n}=12)$ of idarucizumab in patients who had received dabigatran. Participants were enrolled into 1 of 4 dose groups and were randomly assigned 3:1 to idarucizumab or placebo on Day 0. All of the participants were given oral dabigatran at a dose of 220 $\mathrm{mg}$ twice daily for 4 days. Idarucizumab or placebo were then administered 1 hour 55 minutes after the final dose of dabigatran was administered on Day $4 .^{2}$

The second study was a 2-way, cross-over design carried out in 46 participants aged 45-80 years, including healthy male and female volunteers and those with pre-existing mild or moderate kidney impairment (NCT01955720). Dabigatran was administered over 4 days at a dose of 220 $\mathrm{mg}$ twice daily in healthy participants and $150 \mathrm{mg}$ twice daily, also over 4 days, in those with renal impairment. Idarucizumab was given at doses of $1,2.5$, or $5 \mathrm{~g}$, or two

\section{What's new, what's important}

The FDA recently approved idarucizumab for the reversal of the anticoagulant effects of dabigatran in patients taking dabigatran and who need emergency surgery or urgent procedures or face life-threatening or uncontrolled bleeding. Idarucizumab is a monoclonal antibody fragment that binds to the direct thrombin inhibitor dabigatran and reverses its anticoagulant effect. Its approval was based on the findings from 3 randomized, placebo-controlled trials enrolling a total of 283 healthy volunteers who received either dabigatran plus idarucizumab, or dabigatran plus placebo. The findings showed that the anticoagulant effect of dabigatran was completely reversed in more than $89 \%$ of patients within 4 hours of receiving idarucizumab. Elevated coagulation parameters have been observed in a limited number of patients 12-24 hours after idarucizumab administration. The dose is $5 \mathrm{~g}$ IV as 2 consecutive $2.5 \mathrm{~g}$ infusions, or it is given as a bolus by injecting both $2.5-\mathrm{g}$ vials consecutively. Headache was the most common adverse event reported in more than $5 \%$ of the healthy volunteers, other side effects include hypokalemia, delirium, constipation, pyrexia, and pneumonia. This is an important development in the usage of novel anticoagulants and potentially lifesaving for patients on dabigatran.

$$
\text { - Jame Abraham, MD, FACP (abrahaj5@ccf.org) }
$$

$2.5 \mathrm{~g}$ doses given 1 hour apart, and was administered as a 5 -minute IV infusion 2 hours after the last dose of dabigatran. ${ }^{3}$ The third study evaluated single doses of idarucizumab alone and following dabigatran in 80 healthy Japanese subjects (NCT02028780).

The trials demonstrated that idarucizumab alone had no effect on coagulation parameters or endogenous thrombin potential, and that administration of idarucizumab at doses of $2 \mathrm{~g}$ or more resulted in immediate, complete, and sustained reversal of the anticoagulant effects of dabigatran in a dose-dependent manner, as measured by thrombin time, dilute thrombin time, ecarin clotting time, and other assays. The drug was well tolerated with only minor drugrelated effects and no clinically relevant adverse events in healthy volunteers and in those with mild or moderate renal impairment.

The data is further supported by interim results from an

Report prepared by Jane de Lartigue, PhD. JCSO 2016;14:8-10. @2016 Frontline Medical Communications. DOI 10.12788/jcso.0213. 


\section{Mechanism of action: idarucizumab Neutralizing dabigatran's anticoagulant activity}

The coagulation cascade is a biological process by which the blood forms clots to prevent blood loss from damaged vessels that involves activation, adhesion, and aggregation of platelets and deposition of a fibrous protein, fibrin. Following injury, the coagulation response is initiated by 2 different routes, the intrinsic pathway and the extrinsic (or tissue-factor) pathway, which activate a cascade of serine proteases.

The 2 pathways converge into what is dubbed the common pathway at the point where 1 of
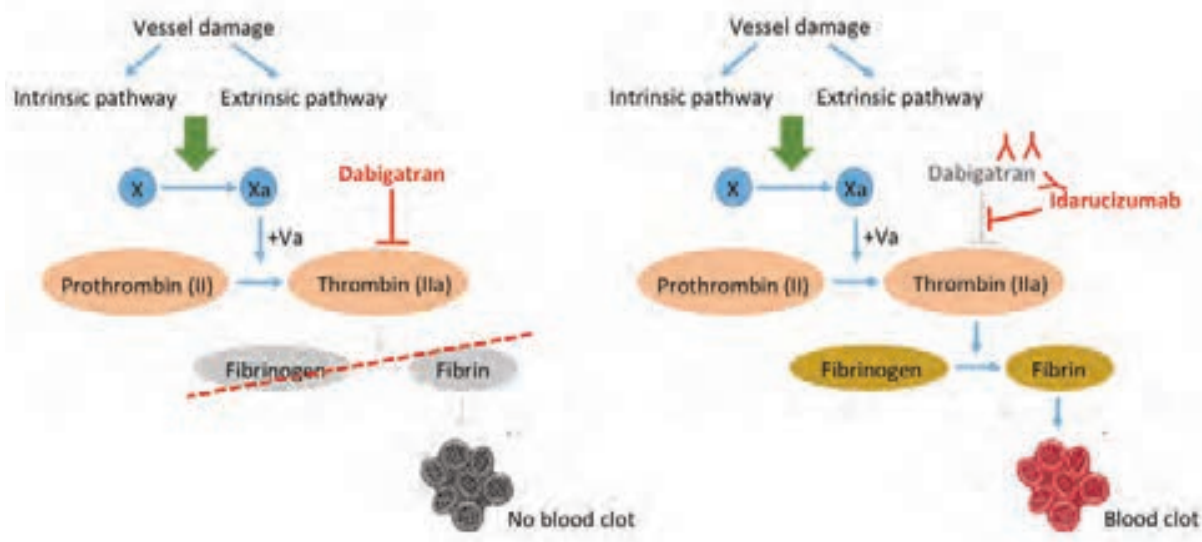

Idarucizumab is a monoclonal antibody fragment that specifically binds to the oral anticoagulant dabigatran and reverses its effects on the coagulation cascade, reinstating a patient's ability to form blood clots.

Illustration: Jane de Lartigue these proteases - Factor $X$ - is

activated to form Factor $\mathrm{Xa}$. In combination with its Cofactor Va, Factor Xa forms the prothrombinase complex that activates prothrombin, leading to the formation of thrombin, the master regulator of the coagulation cascade. Thrombin converts soluble fibrinogen into insoluble fibrin and activates the surrounding platelets, which together generate a hemostatic plug (otherwise known as a blood clot) that seals the breach in the vessel.

Anticoagulants are a class of drug that has been developed to prevent the clotting of blood, for the treatment of thrombotic disorders - medical conditions characterized by abnormal clots and emboli. Traditional anticoagulants include heparin and vitamin $\mathrm{K}$ antagonists. Given the nature of their mechanism of action, these drugs can potentially lead to unanticipated severe bleeding or enhanced risk of bleeding, particularly in the setting of medical trauma or illness or in patients undergoing invasive surgical procedures. For this reason, they have been developed in conjunction with antidotes that can reverse their effects when needed.

In the past several decades, newer oral anticoagulants have been developed that directly inhibit components of the coagulation cascade, among them, dabigatran is a direct thrombin inhibitor. Historically, these drugs were used without the availability of specific antidotes, however, the approval of idarucizumab marks the availability of the first such reversal agent. Idarucizumab is a humanized monoclonal antibody fragment, produced in a recombinant mammalian cell line, which specifically binds to dabigatran and prevents it from binding to thrombin. This neutralizes the anticoagulant effects of dabigatran and reinstates the coagulation cascade so that a patient's blood can clot again. ongoing, open-label phase 3 trial. ${ }^{4}$ REVERSE-AD is a prospective cohort study in patients with serious bleeding (Group A) or those who require urgent surgery (Group B) that aims to enroll 123 patients. The primary endpoint will be the maximum percentage reversal of dabigatran's anticoagulant effect, as measured by ecarin clotting time or dilute thrombin time, within 4 hours after the administration of 5 $\mathrm{g}$ idarucizumab. A key secondary endpoint is restoration of hemostasis. The interim analysis has detailed results from 90 patients (51 from Group A, 39 from Group B).

Among 68 patients with elevated dilute thrombin time and 81 patients with elevated ecarin clotting time at baseline, the median maximum percentage reversal was $100 \%$. Idarucizumab normalized clotting times in $88 \%-98 \%$ of patients within minutes, and they remained normal for the subsequent 24 hours. Intraoperative hemostasis was normal among 33 of 36 patients who underwent a surgical procedure, and only mildly or moderately impaired in the remaining 3 . In patients with major bleeding, hemostasis was restored at a median of 11.4 hours.

There were no safety concerns at interim analysis. Eighteen participants died -9 in each study group; 10 deaths were from vascular causes, including 5 fatal bleeding events. Deaths within 96 hours after treatment seemed to be related to the index event, whereas later deaths were associated with coexisting conditions. Thrombotic events occurred in 5 patients, and none of those patients were receiving antithrombotic therapy when the events occurred. Overall, 21 patients (13 in Group A, 8 in Group B) experienced serious adverse events, including gastrointestinal hemorrhage and postoperative wound infection, delirium, right ventricular failure, and pulmonary edema. 


\section{Community Translations}

The prescribing information ${ }^{5}$ for idarucizumab, which is marketed as Praxbind by Boehringer Ingelheim, recommends a dose of $5 \mathrm{~g}$ idarucizumab and details warnings and precautions relating to thromboembolic risk, re-elevation of coagulation parameters (eg, activated partial thromboplastin time or ecarin clotting time), hypersensitivity reactions, and risk of serious adverse reactions in patients with hereditary fructose intolerance. In the event of a thromboembolic event, it is recommended that anticoagulant therapy

\section{References}

1. Glund S, Moschetti V, Norris S, et al. A randomized study in healthy volunteers to investigate the safety, tolerability and pharmacokinetics of idarucizumab, a specific antidote to dabigatran. Thromb Hemost. 2015;113:943-951.

2. Glund S, Stangier J, Schmohl M, et al. Safety, tolerability, and efficacy of idarucizumab for the reversal of the anticoagulant effect of dabigatran in healthy male volunteers: a randomized, placebo-controlled, double-blind phase 1 trial. Lancet. 2015;386:680-690.

3. Glund S, Stangier J, Schmohl M, et al. Idarucizumab, a specific antidote for dabigatran: immediate, complete and sustained reversal of be resumed as soon as medically possible. Administration of an additional $5 \mathrm{~g}$ idarucizumab may be considered in the case of re-elevated coagulation parameters. If anaphylactic reactions or other serious reactions occur, they should be treated appropriately and idarucizumab discontinued immediately. Idarucizumab contains $4 \mathrm{~g}$ sorbitol as an excipient and this should be taken into consideration as part of the combined metabolic load of sorbitol/fructose in patients with hereditary fructose intolerance.

dabigatran induced anticoagulation in elderly and renally impaired subjects. Blood. 2014;124:344-353.

4. Pollack Jr CV, Reilly PA, Eikelboom J, et al. Idarucizumab for dabigatran reversal. N Engl J Med. 2015;373:511-520.

5. Praxbind (idarucizumab). Prescribing information. Ridgefield, CT: Boehringer Ingelheim Pharmaceuticals Inc. http://us.boehringeringelheim.com/content/dam/internet/opu/us_EN/documents/ Media_Press_Releases/2015/Praxbind.pdf. Revised October 2015. Accessed November 21, 2015. 